\title{
LARGE CLIQUES IN A POWER-LAW RANDOM GRAPH
}

\author{
SVANTE JANSON, ${ }^{*}$ Uppsala University \\ TOMASZ ŁUCZAK, ${ }^{* *}$ Adam Mickiewicz University \\ ILKKA NORROS, ${ }^{* * *}$ VTT Technical Research Centre of Finland
}

\begin{abstract}
In this paper we study the size of the largest clique $\omega(G(n, \alpha))$ in a random graph $G(n, \alpha)$ on $n$ vertices which has power-law degree distribution with exponent $\alpha$. We show that, for 'flat' degree sequences with $\alpha>2$, with high probability, the largest clique in $G(n, \alpha)$ is of a constant size, while, for the heavy tail distribution, when $0<\alpha<2, \omega(G(n, \alpha))$ grows as a power of $n$. Moreover, we show that a natural simple algorithm with high probability finds in $G(n, \alpha)$ a large clique of size $(1-o(1)) \omega(G(n, \alpha))$ in polynomial time.
\end{abstract}

Keywords: Power-law random graph; clique; greedy algorithm

2010 Mathematics Subject Classification: Primary 05C80

Secondary 05C69; 60C05

\section{Introduction}

Random graphs with finite density and power-law degree sequence have attracted much attention in the last few years (see, e.g. [8] and the references therein). Several models for such graphs have been proposed; in this paper we concentrate on a Poissonian model $G(n, \alpha)$ in which the number of vertices of degree at least $i$ decreases roughly as $n i^{-\alpha}$ (for a precise definition of the model, see Section 2).

We show (Theorem 1) that there is a major difference in the size of the largest clique $\omega(G(n, \alpha))$ between the cases $\alpha<2$ and $\alpha>2$ with an intermediate result for $\alpha=2$. In the 'light tail case', when $\alpha>2$ (this is when the asymptotic degree distribution has a finite second moment), the size of the largest clique is either two or three, i.e. it is almost the same as in the standard binomial model of the random graph $G(n, p)$ in which the expected average degree is a constant. On the other hand, in the 'heavy tail case', when $0<\alpha<2, \omega(G(n, \alpha))$ grows roughly as $n^{1-\alpha / 2}$. In the critical case when $\alpha=2$ we have $\omega(G(n, \alpha))=O_{\mathrm{p}}(1)$, but the probability that $G(n, \alpha) \geq k$ is bounded away from 0 for every $k$. We also show (Corollary 1 ) that in each of the above cases there exists a simple algorithm which, with high probability (w.h.p.), finds in $G(n, \alpha)$ a clique of size $(1-o(1)) \omega(G(n, \alpha))$. This is quite different from the binomial case, where it is widely believed that finding a large clique is hard (see, e.g. [10]).

\footnotetext{
Received 5 May 2009; revision received 10 June 2010.

* Postal address: Department of Mathematics, Uppsala University, PO Box 480, SE-751 06 Uppsala, Sweden.

Email address: svante.janson@math.uu.se

** Postal address: Faculty of Mathematics and Computer Science, Adam Mickiewicz University, ul. Umultowska 87,

61-614 Poznań, Poland. Email address: tomasz@amu.edu.pl

*** Postal address: VTT Technical Research Centre of Finland, PO Box 1000, 02044 VTT, Finland.

Email address: ilkka.norros@vtt.fi
} 
Similar but less precise results have been obtained by Bainconi and Marsili [1], [2] for a slightly different model (see Subsection 6.6). The existence of large cliques in random graphs with a heavy tail degree sequence has also been observed by van der Hofstad et al. [16].

\section{The model and the results}

The model we study is a modification of the conditionally Poissonian random graph studied in [14] (the significance of this modification will be studied in Subsection 6.5; see also [7] for a related model). For $\alpha>1$, it is also an example of the 'rank 1 case' of an inhomogeneous random graph studied in [5, Section 16.4].

In order to define our model, consider a set of $n$ vertices (labelled $1, \ldots, n$ for convenience). We first assign a capacity or weight $W_{i}$ to each vertex $i$. For definiteness and simplicity, we assume that these are independent and identically distributed random variables with a distribution with a power-law tail

$$
\mathrm{P}(W>x)=a x^{-\alpha}, \quad x \geq x_{0},
$$

for some constants $a>0$ and $\alpha>0$, and some $x_{0}>0$ (here and below, we write $W$ for any of the $W_{i}$ when the index does not matter). Thus, for example, $W$ could have a Pareto distribution, when $x_{0}=a^{1 / \alpha}$ and $\mathrm{P}(W>x)=1$ for $x \leq x_{0}$, but the distribution could be arbitrarily modified for small $x$. We denote the largest weight by $W_{\max }=\max _{i} W_{i}$. Observe that (1) implies that

$$
\mathrm{P}\left(W_{\max }>t n^{1 / \alpha}\right) \leq n \mathrm{P}\left(W>t n^{1 / \alpha}\right)=O\left(t^{-\alpha}\right) .
$$

Note also that $\mathrm{E} W^{\beta}<\infty$ if and only if $\alpha>\beta$; in particular, for the 'heavy tail case' when $\alpha \leq 2$, we have $\mathrm{E} W^{2}=\infty$.

Now, conditionally given the weights $\left\{W_{i}\right\}_{1}^{n}$, we join each pair $\{i, j\}$ of vertices by $E_{i j}$ parallel edges, where the numbers $E_{i j}$ are independent Poisson-distributed random numbers with means

$$
\mathrm{E} E_{i j}=\lambda_{i j}=b \frac{W_{i} W_{j}}{n},
$$

where $b>0$ is another constant. We denote the resulting random (multi)graph by $\hat{G}(n, \alpha)$. For our purposes, parallel edges can be merged into a single edge, so we may alternatively define $G(n, \alpha)$ as the random simple graph where vertices $i$ and $j$ are joined by an edge with probability

$$
p_{i j}=1-\exp \left(-\lambda_{i j}\right)
$$

independently for all pairs $(i, j)$ with $1 \leq i<j \leq n$.

Then our main result can be stated as follows. Let us recall that an event holds w.h.p. if it holds with probability tending to 1 as $n \rightarrow \infty$. We also use $o_{\mathrm{p}}$ and $O_{\mathrm{p}}$ in the standard sense (see, e.g. [12, pp. 10-11]).

Theorem 1. (i) If $0<\alpha<2$ then

$$
\omega(G(n, \alpha))=\left(c+o_{\mathrm{p}}(1)\right) n^{1-\alpha / 2}(\log n)^{-\alpha / 2},
$$

where

$$
c=a b^{\alpha / 2}\left(1-\frac{\alpha}{2}\right)^{-\alpha / 2} .
$$


(ii) If $\alpha=2$ then $\omega(G(n, \alpha))=O_{\mathrm{p}}(1)$; that is, for every $\varepsilon>0$, there exists a constant $C_{\varepsilon}$ such that, for every $n, \mathrm{P}\left(\omega(G(n, \alpha))>C_{\varepsilon}\right) \leq \varepsilon$. However, there is no fixed finite bound $C$ such that $\omega(G(n, \alpha)) \leq C$ w.h.p.

(iii) If $\alpha>2$ then $\omega(G(n, \alpha)) \in\{2,3\}$ w.h.p. Moreover, the probabilities of each of the events $\omega(G(n, \alpha))=2$ and $\omega(G(n, \alpha))=3$ tend to positive limits, given by (24) below.

A question which naturally emerges when studying the size of the largest clique in a class of graphs is whether we can find a large clique in such graphs in a polynomial time. By Theorem 1 , w.h.p., we can find $\omega(G(n, \alpha))$ in a polynomial time for $\alpha>2$, and, with some extra effort, the same can be accomplished for $\alpha=2$ (see Corollary 1). Thus, we concentrate on the case in which $\alpha<2$, when the large clique is of polynomial size. Let us suppose that we know the vertex weights $W_{i}$ defined in Section 2 and, for simplicity, that these are distinct (otherwise we resolve ties randomly; we omit the details). Since vertices with larger weights tend to have higher degrees, they are more likely to be in a large clique, so it is natural to try to find a large clique by looking at the vertices with largest weights. One simple way is the greedy algorithm which checks the vertices in order of decreasing weights and selects every vertex that is joined to every other vertex already selected. This evidently yields a clique, which we call the greedy clique and denote by $\mathcal{K}_{\text {gr }}$. Thus,

$$
\mathcal{K}_{\mathrm{gr}}=\left\{i: i \sim j \text { for all } j \text { with } W_{j}>W_{i} \text { and } j \in \mathcal{K}_{\mathrm{gr}}\right\} .
$$

A simplified algorithm is to select every vertex that is joined to every vertex with higher weight, regardless of whether these are already selected or not. This gives the quasi top clique studied in [13], which we denote by $\mathcal{K}_{\mathrm{qt}}$. Thus,

$$
\mathcal{K}_{\mathrm{qt}}=\left\{i: i \sim j \text { for all } j \text { with } W_{j}>W_{i}\right\} .
$$

Obviously, $\mathcal{K}_{\mathrm{qt}} \subseteq \mathcal{K}_{\mathrm{gr}}$. The difference between the two cliques is that if we, while checking vertices in order of decreasing weights, reject a vertex, then that vertex is ignored for future tests when constructing $\mathcal{K}_{\mathrm{gr}}$, but not for $\mathcal{K}_{\mathrm{qt}}$. A more drastic approach is to stop at the first failure; we define the full top clique, $\mathcal{K}_{\mathrm{ft}}$, as the result, i.e.

$$
\mathcal{K}_{\mathrm{ft}}=\left\{i: j \sim k \text { for all distinct } j, k \text { with } W_{j}, W_{k} \geq W_{i}\right\} .
$$

Thus, $\mathcal{K}_{\mathrm{ft}}$ is the largest clique consisting of all vertices with weights in some interval $[w, \infty)$. Clearly, $\mathcal{K}_{\mathrm{ft}} \subseteq \mathcal{K}_{\mathrm{qt}} \subseteq \mathcal{K}_{\mathrm{gr}}$. Finally, by $\mathcal{K}_{\max }$ we denote the largest clique (chosen at random, say, if there is a tie). Thus,

$$
\left|\mathcal{K}_{\mathrm{ft}}\right| \leq\left|\mathcal{K}_{\mathrm{qt}}\right| \leq\left|\mathcal{K}_{\mathrm{gr}}\right| \leq\left|\mathcal{K}_{\max }\right|=\omega(G(n, \alpha)) .
$$

The following theorem shows that the last two inequalities in (6) are asymptotic equalities, but not the first one. Here, we use ' $\stackrel{\text { P }}{\rightarrow}$ ' for convergence in probability, and all unspecified limits are as $n \rightarrow \infty$.

Theorem 2. For $G(n, \alpha)$ with $0<\alpha<2$, both $\mathcal{K}_{\mathrm{gr}}$ and $\mathcal{K}_{\mathrm{qt}}$ have size $\left(1-o_{\mathrm{p}}(1)\right) \omega(G(n, \alpha))$; in other words,

On the other hand,

$$
\frac{\left|\mathcal{K}_{\mathrm{gr}}\right|}{\left|\mathcal{K}_{\max }\right|} \stackrel{\mathrm{P}}{\rightarrow} 1 \text { and } \frac{\left|\mathcal{K}_{\mathrm{qt}}\right|}{\left|\mathcal{K}_{\max }\right|} \stackrel{\mathrm{P}}{\rightarrow} 1 \text {. }
$$

$$
\frac{\left|\mathcal{K}_{\mathrm{ft}}\right|}{\left|\mathcal{K}_{\max }\right|} \stackrel{\mathrm{P}}{\rightarrow} 2^{-\alpha / 2} .
$$


Thus, w.h.p., $\mathcal{K}_{\text {gr }}$ and $\mathcal{K}_{\mathrm{qt}}$ almost attain the maximum size of a clique, while $\mathcal{K}_{\mathrm{ft}}$ falls short by a constant factor. As a simple corollary of the above result, we obtain the following.

Corollary 1. For every $\alpha>0$, there exists an algorithm which, w.h.p., finds in $G(n, \alpha)$ a clique of size $(1-o(1)) \omega(G(n, \alpha))$ in a polynomial time.

\section{The proof for the case $\alpha<2$ (no second moment)}

We begin with a simple lemma giving an upper bound for the clique number of the ErdôsRényi random graph $G(n, p)$ (for much more precise results, see, e.g. [12]).

Lemma 1. For any $p=p(n)$, w.h.p.,

$$
\omega(G(n, p)) \leq \frac{2 \log n}{1-p} .
$$

Proof. Denote by $X_{k}$ the number of cliques of size $k$ in $G(n, p)$. For the expected number of such cliques, we have

$$
\mathrm{E} X_{k}=\left(\begin{array}{l}
n \\
k
\end{array}\right) p^{\left(\begin{array}{l}
k \\
2
\end{array}\right)} \leq\left(\frac{n \mathrm{e}}{k} p^{(k-1) / 2}\right)^{k}
$$

If we set $k \geq\lfloor 2 \log (n) /(1-p)\rfloor$ then

$$
p^{(k-1) / 2}=(1-(1-p))^{(k-1) / 2} \leq \mathrm{e}^{-(1-p)(k-1) / 2} \leq \frac{\mathrm{e}}{n} .
$$

Consequently, we arrive at

$$
\mathrm{P}(\omega(G(n, p)) \geq k)=\mathrm{P}\left(X_{k} \geq 1\right) \leq \mathrm{E} X_{k} \leq\left(\frac{\mathrm{e}^{2}}{k}\right)^{k} \rightarrow 0,
$$

since $k \geq\lfloor 2 \log n\rfloor \rightarrow \infty$.

Proofs of Theorems $1(i)$ and 2. For $s>0$, let us partition the vertex set $V=\{1, \ldots, n\}$ of $G_{n}=G(n, \alpha)$ into

$$
V_{s}^{-}=\left\{i: W_{i} \leq s \sqrt{n \log n}\right\} \quad \text { and } \quad V_{s}^{+}=\left\{i: W_{i}>s \sqrt{n \log n}\right\}
$$

we may think of elements of $V_{s}^{-}$and $V_{s}^{+}$as 'light' and 'heavy' vertices, respectively. By (1),

$$
\mathrm{E}\left|V_{s}^{+}\right|=n \mathrm{P}(W>s \sqrt{n \log n})=a s^{-\alpha} n^{1-\alpha / 2} \log ^{-\alpha / 2} n .
$$

Moreover, $\left|V_{s}^{+}\right| \sim \operatorname{Bin}(n, \mathrm{P}(W>s \sqrt{n \log n}))$, and Chebyshev's inequality (or the sharper Chernoff bounds [12, Section 2.1]) implies that, w.h.p.,

$$
\left|V_{s}^{+}\right|=(1+o(1)) \mathrm{E}\left|V_{s}^{+}\right|=(1+o(1)) a s^{-\alpha} n^{1-\alpha / 2} \log ^{-\alpha / 2} n .
$$

We now condition on the sequence of weights $\left\{W_{k}\right\}$. We will repeatedly use the fact that if $i$ and $j$ are vertices with weights $W_{i}=x \sqrt{n \log n}$ and $W_{j}=y \sqrt{n \log n}$, then, by (3) and (4), $\lambda_{i j}=b x y \log n$ and

$$
p_{i j}=1-\mathrm{e}^{-\lambda_{i j}}=1-\mathrm{e}^{-b x y \log n}=1-n^{-b x y} .
$$


In particular,

$$
\begin{array}{ll}
p_{i j} \leq 1-n^{-b s^{2}} & \text { if } i, j \in V_{s}^{-}, \\
p_{i j}>1-n^{-b s^{2}} & \text { if } i, j \in V_{s}^{+} .
\end{array}
$$

Consider, still conditioning on $\left\{W_{k}\right\}$, for an $s$ that will be chosen later, the induced subgraph $G_{n}\left[V_{s}^{-}\right]$of $G(n, \alpha)$ with vertex set $V_{s}^{-}$. This graph has at most $n$ vertices and, by (9), all edge probabilities are at most $1-n^{-b s^{2}}$, so we may regard $G_{n}\left[V_{s}^{-}\right]$as a subgraph of $G(n, p)$ with $p=1-n^{-b s^{2}}$. Hence, Lemma 1 implies that, w.h.p.,

$$
\omega\left(G_{n}\left[V_{s}^{-}\right]\right) \leq \frac{2 \log n}{n^{-b s^{2}}}=2 n^{b s^{2}} \log n .
$$

If $\mathcal{K}$ is any clique in $G(n, \alpha)$ then $\mathcal{K} \cap V_{s}^{-}$is a clique in $G_{n}\left[V_{s}^{-}\right]$, and, thus, $\left|\mathcal{K} \cap V_{s}^{-}\right| \leq$ $\omega\left(G_{n}\left[V_{s}^{-}\right]\right)$; furthermore, trivially, $\left|\mathcal{K} \cap V_{s}^{+}\right| \leq\left|V_{s}^{+}\right|$. Hence, $|\mathcal{K}| \leq \omega\left(G_{n}\left[V_{s}^{-}\right]\right)+\left|V_{s}^{+}\right|$, and, thus,

$$
\omega(G(n, \alpha)) \leq \omega\left(G_{n}\left[V_{s}^{-}\right]\right)+\left|V_{s}^{+}\right| .
$$

We choose, for a given $\varepsilon>0, s=(1-\varepsilon) b^{-1 / 2}(1-\alpha / 2)^{1 / 2}$ so that the exponents of $n$ in (7) and (11) almost match; we then obtain, from (12), (11), and (7), that, w.h.p.,

$$
\begin{aligned}
\omega(G(n, \alpha)) & \leq(1+o(1)) a s^{-\alpha} n^{1-\alpha / 2} \log ^{-\alpha / 2} n \\
& =(1+o(1))(1-\varepsilon)^{-\alpha} c n^{1-\alpha / 2} \log ^{-\alpha / 2} n,
\end{aligned}
$$

with $c$ defined as in (5).

To obtain a matching lower bound, we consider the quasi top clique $\mathcal{K}_{\mathrm{qt}}$. Again, let $s$ be fixed and condition on the weights $\left\{W_{k}\right\}$. If $i, j \in V_{s}^{+}$then, by (10), the probability that $i$ is not joined to $j$ is less than $n^{-b s^{2}}$. Hence, conditioned on the weights $\left\{W_{k}\right\}$, the probability that a given vertex $i \in V_{s}^{+}$is not joined to every other $j \in V_{s}^{+}$is at most $\left|V_{s}^{+}\right| n^{-b s^{2}}$, which, by (7), w.h.p., is at most $2 a s^{-\alpha} n^{1-\alpha / 2-b s^{2}} \log ^{-\alpha / 2} n$. We now choose $s=(1+\varepsilon) b^{-1 / 2}(1-\alpha / 2)^{1 / 2}$ with $\varepsilon>0$. Then, for some constant $C<\infty$, w.h.p.,

$$
\mathrm{P}\left(i \notin \mathcal{K}_{\mathrm{qt}} \mid\left\{W_{k}\right\}\right) \leq C n^{-2 \varepsilon(1-\alpha / 2)},
$$

and, thus,

$$
\mathrm{E}\left(\left|V_{s}^{+} \backslash \mathcal{K}_{\mathrm{qt}}\right| \mid\left\{W_{k}\right\}\right) \leq C n^{-2 \varepsilon(1-\alpha / 2)}\left|V_{s}^{+}\right| .
$$

Hence, by Markov's inequality, w.h.p.,

$$
\left|V_{s}^{+} \backslash \mathcal{K}_{\mathrm{qt}}\right| \leq C n^{-\varepsilon(1-\alpha / 2)}\left|V_{s}^{+}\right|
$$

Thus, using (7), w.h.p.,

$$
\begin{aligned}
\omega(G(n, \alpha)) & \geq\left|\mathcal{K}_{\mathrm{gr}}\right| \\
& \geq\left|\mathcal{K}_{\mathrm{qt}}\right| \\
& \geq\left|V_{s}^{+}\right|-\left|V_{s}^{+} \backslash \mathcal{K}_{\mathrm{qt}}\right| \\
& \geq(1-o(1))\left|V_{s}^{+}\right| \\
& \geq(1-o(1)) a s^{-\alpha} n^{1-\alpha / 2} \log ^{-\alpha / 2} n \\
& =(1-o(1))(1+\varepsilon)^{-\alpha} c n^{1-\alpha / 2} \log ^{-\alpha / 2} n .
\end{aligned}
$$

Since $\varepsilon>0$ is arbitrary, (13) and (14) imply Theorem 1(i) and the first part of Theorem 2. 
In order to complete the proof of Theorem 2, it remains to consider $\mathcal{K}_{\mathrm{ft}}$. Define $\bar{G}_{n}$ as the complement of $G(n, \alpha)$. Then, using (10) and conditioned on $\left\{W_{i}\right\}$, we infer that the expected number of edges of $\bar{G}_{n}$ with both endpoints in $V_{s}^{+}$is at most $n^{-b s^{2}}\left|V_{s}^{+}\right|^{2}$. If we choose $s=b^{-1 / 2}(2-\alpha)^{1 / 2}$ then (7) implies that this is, w.h.p., $o(1)$; hence, w.h.p., $V_{s}^{+}$contains no edges of $\bar{G}_{n}$, i.e. $\mathcal{K}_{\mathrm{ft}} \supseteq V_{s}^{+}$.

On the other hand, let $0<\varepsilon<\frac{1}{2}$ and define, still with $s=b^{-1 / 2}(2-\alpha)^{1 / 2}$,

$$
V^{\prime}=V_{(1-2 \varepsilon) s}^{+} \cap V_{(1-\varepsilon) s}^{-} .
$$

Then, conditioned on $\left\{W_{i}\right\}$, the probability of having no edges of $\bar{G}_{n}$ in $V^{\prime}$ is, by (9),

$$
\left.\prod_{i, j \in V^{\prime}, i<j} p_{i j} \leq\left(1-n^{-b(1-\varepsilon)^{2} s^{2}}\right)^{\left(V^{\prime} \mid\right.}\right) \leq \exp \left(-\frac{1}{2} n^{-(1-\varepsilon)^{2}(2-\alpha)}\left(\left|V^{\prime}\right|-1\right)^{2}\right)
$$

By (7), w.h.p.,

$$
\begin{aligned}
\left|V^{\prime}\right|-1 & =\left|V_{(1-2 \varepsilon) s}^{+}\right|-\left|V_{(1-\varepsilon) s}^{+}\right|-1 \\
& =(1+o(1)) a\left((1-2 \varepsilon)^{-\alpha}-(1-\varepsilon)^{-\alpha}\right) s^{-\alpha} n^{1-\alpha / 2} \log ^{-\alpha / 2} n,
\end{aligned}
$$

and it follows from (15) that

$$
\mathrm{P}\left(\mathcal{K}_{\mathrm{ft}} \supseteq V_{(1-2 \varepsilon) s}^{+}\right) \leq \prod_{i, j \in V^{\prime}, i<j} p_{i j} \rightarrow 0 .
$$

Hence, w.h.p., $\mathcal{K}_{\mathrm{ft}} \subset V_{(1-2 \varepsilon) s}^{+}$.

We have shown that, for any $\varepsilon \in\left(0, \frac{1}{2}\right)$, w.h.p., $V_{s}^{+} \subseteq \mathcal{K}_{\mathrm{ft}} \subset V_{(1-2 \varepsilon) s}^{+}$, and it follows, by (5) and (7) (by letting $\varepsilon \rightarrow 0$ ), that, w.h.p.,

$$
\begin{aligned}
\left|\mathcal{K}_{\mathrm{ft}}\right| & =(1+o(1))\left|V_{s}^{+}\right| \\
& =(1+o(1)) a s^{-\alpha} n^{1-\alpha / 2} \log ^{-\alpha / 2} n \\
& =(1+o(1)) 2^{-\alpha / 2} c n^{1-\alpha / 2} \log ^{-\alpha / 2} n \\
& =(1+o(1)) 2^{-\alpha / 2} \omega(G(n, \alpha)),
\end{aligned}
$$

where the last equality follows from Theorem 1.

\section{The case $\alpha=2$ (still no second moment)}

Proofs of Theorem 1(ii) and Corollary 1. Given the weights $W_{i}$, the probability that four vertices $i, j, k$, and $l$ form a clique is, by (3) and (4),

$$
p_{i j} p_{i k} p_{i l} p_{j k} p_{j l} p_{k l} \leq \lambda_{i j} \lambda_{i k} \lambda_{i l} \lambda_{j k} \lambda_{j l} \lambda_{k l}=b^{6} \frac{W_{i}^{3} W_{j}^{3} W_{k}^{3} W_{l}^{3}}{n^{6}} .
$$

Thus, if $X_{m}$ is the number of cliques of size $m$ in $G(n, \alpha)$ then the conditional expectation of $X_{4}$ is

$$
\mathrm{E}\left(X_{4} \mid\left\{W_{i}\right\}_{1}^{n}\right) \leq b^{6} n^{-6} \sum_{i<j<k<l} W_{i}^{3} W_{j}^{3} W_{k}^{3} W_{l}^{3} \leq b^{6}\left(n^{-3 / 2} \sum_{i} W_{i}^{3}\right)^{4} .
$$


To show that the number of such cliques of size 4 is bounded in probability, we shall calculate a truncated expectation of $\sum_{i} W_{i}^{3}$. Using (1), for any constant $A>0$, we obtain

$$
\begin{aligned}
\mathrm{E}\left(\sum_{i} W_{i}^{3} ; W_{\max } \leq A n^{1 / 2}\right) & \leq \mathrm{E}\left(\sum_{i} \min \left(W_{i}, A n^{1 / 2}\right)^{3}\right) \\
& =n \mathrm{E} \min \left(W, A n^{1 / 2}\right)^{3} \\
& =n \int_{0}^{A n^{1 / 2}} 3 x^{2} \mathrm{P}(W>x) \mathrm{d} x \\
& =O\left(n A n^{1 / 2}\right),
\end{aligned}
$$

and, thus, using (2) and Markov's inequality, for every $t>0$ and some constant $C$ independent of $A, t$, and $n$, we infer that

$$
\begin{aligned}
\mathrm{P}\left(n^{-3 / 2} \sum_{i} W_{i}^{3}>t\right) & \leq t^{-1} \mathrm{E}\left(n^{-3 / 2} \sum_{i} W_{i}^{3} ; W_{\max } \leq A n^{1 / 2}\right)+\mathrm{P}\left(W_{\max }>A n^{1 / 2}\right) \\
& \leq C A t^{-1}+C A^{-2} .
\end{aligned}
$$

Given $t>0$, we choose $A=t^{1 / 3}$ and find that $\mathrm{P}\left(n^{-3 / 2} \sum_{i} W_{i}^{3}>t\right)=O\left(t^{-2 / 3}\right)$. Hence, $n^{-3 / 2} \sum_{i} W_{i}^{3}=O_{\mathrm{p}}(1)$, and it follows, by (16) and Markov's inequality, that $X_{4}=O_{\mathrm{p}}(1)$.

Finally, we observe that, for any $m \geq 4$,

$$
\mathrm{P}(\omega(G(n, \alpha)) \geq m) \leq \mathrm{P}\left(X_{4} \geq\left(\begin{array}{c}
m \\
4
\end{array}\right)\right),
$$

which can thus be made arbitrarily small (uniformly in $n$ ) by choosing large enough $m$. Hence, $\omega(G(n, \alpha))=O_{\mathrm{p}}(1)$.

To complete the proof of Theorem 1(ii), let us note that, for any fixed $m \leq n$, the probability that there are at least $m$ vertices with weights $W_{i}>n^{1 / 2}$ is larger than $c_{1}>\overline{0}$ for some absolute constant $c_{1}>0$, and conditioned on this event, the probability that the first $m$ of these vertices form a clique is larger than $c_{2}$ for some absolute constants $c_{1}, c_{2}$ not depending on $n$.

Finally, we remark that all cliques of size 4 can clearly be found in time $O\left(n^{4}\right)$. The number of such cliques is, w.h.p., at most $\log \log n$, say, so there exists an algorithm which, w.h.p., finds the largest clique in a polynomial time (for example, by crudely checking all sets of cliques of size 4).

\section{The case $\alpha>2$ (finite second moment)}

Proof of Theorem 1(iii). Choose $v$ such that $\frac{1}{2}>v>1 / \alpha$. Then (2) (or (1) directly) implies that, w.h.p., $W_{\max } \leq n^{\nu}$. Furthermore, in analogy to (17) and (18),

$$
\begin{aligned}
\mathrm{E}\left(\sum_{i} W_{i}^{3} ; W_{\max } \leq n^{\nu}\right) & \leq \mathrm{E}\left(\sum_{i} \min \left(W_{i}, n^{\nu}\right)^{3}\right) \\
& =n \int_{0}^{n^{v}} 3 x^{2} \mathrm{P}(W>x) \mathrm{d} x \\
& =O\left(n n^{\nu}\right) \\
& =o\left(n^{3 / 2}\right)
\end{aligned}
$$


and, thus,

$$
\begin{aligned}
\mathrm{P}\left(n^{-3 / 2} \sum_{i} W_{i}^{3}>t\right) & \leq t^{-1} \mathrm{E}\left(n^{-3 / 2} \sum_{i} W_{i}^{3} ; W_{\max } \leq n^{v}\right)+\mathrm{P}\left(W_{\max }>n^{\nu}\right) \\
& =o(1) .
\end{aligned}
$$

Hence, $n^{-3 / 2} \sum_{i} W_{i}^{3} \stackrel{\mathrm{P}}{\rightarrow} 0$, and it follows from (16) that

$$
\mathrm{P}\left(X_{4} \geq 1 \mid\left\{W_{i}\right\}_{1}^{n}\right) \leq \mathrm{E}\left(X_{4} \mid\left\{W_{i}\right\}_{1}^{n}\right) \stackrel{\mathrm{P}}{\rightarrow} 0 .
$$

Dominated convergence yields

$$
\mathrm{P}(\omega(G(n, \alpha)) \geq 4)=\mathrm{P}\left(X_{4} \geq 1\right)=\mathrm{EP}\left(X_{4} \geq 1 \mid\left\{W_{i}\right\}_{1}^{n}\right) \rightarrow 0 .
$$

Consequently, w.h.p., $\omega(G(n, \alpha)) \leq 3$.

Moreover, for the number, $X_{3}$, of cliques of size 3 , we have

$$
\mathrm{E} X_{3} \leq \mathrm{E} \sum_{i<j<k} \lambda_{i j} \lambda_{i k} \lambda_{j k}=\mathrm{E}\left(b^{3} n^{-3} \sum_{i<j<k} W_{i}^{2} W_{j}^{2} W_{k}^{2}\right) \leq \frac{1}{6} b^{3}\left(\mathrm{E} W^{2}\right)^{3} .
$$

Note that $\mathrm{E} W^{2}<\infty$ by (1) and the assumption that $\alpha>2$. Hence, the number of $K_{3}$ in $G(n, \alpha)$ is $O_{\mathrm{p}}(1)$. To obtain the limit distribution, it is convenient to truncate the distribution, as we have done in the previous section. Let $A$ be a fixed large constant, let $X_{3}^{A}$ be the number of $K_{3}$ in $G(n, \alpha)$ such that all three vertices have weights at most $A$, and let $X_{3}^{A *}$ be the number of the remaining triangles. Arguing as in (20), we easily see that

$$
\begin{aligned}
\mathrm{E} X_{3}^{A} & \leq \frac{1}{6} b^{3}\left(\mathrm{E}\left(W^{2} ; W \leq A\right)\right)^{3}, \\
\mathrm{E} X_{3}^{A *} & \leq b^{3}\left(\mathrm{E}\left(W^{2}\right)\right)^{2} \mathrm{E}\left(W^{2} ; W>A\right) .
\end{aligned}
$$

Moreover, if $W_{i}, W_{j} \leq A$ then $\lambda_{i j}=O(1 / n)$, and, thus, $p_{i j} \sim \lambda_{i j}$. It is easily seen that (21) can be sharpened to

$$
\mathrm{E} X_{3}^{A} \rightarrow \mu_{A}=\frac{1}{6} b^{3}\left(\mathrm{E}\left(W^{2} ; W \leq A\right)\right)^{3} .
$$

Furthermore, we may calculate fractional moments $\mathrm{E}\left(X_{3}^{A}\right)_{m}$ by the same method, and it follows easily by a standard argument (see, e.g. [12, Theorem 3.19] for $G(n, p)$ ) that $\mathrm{E}\left(X_{3}^{A}\right)_{m} \rightarrow \mu_{A}^{m}$ for every $m \geq 1$. Thus, by the method of moments [12, Corollary 6.8],

$$
X_{3}^{A} \stackrel{\mathrm{D}}{\rightarrow} \operatorname{Po}\left(\mu_{A}\right)
$$

as $n \rightarrow \infty$ for every fixed $A$, where $\stackrel{\text { ' }}{\rightarrow}$ ' denotes convergence in distribution.

To conclude the proof, observe that the right-hand side of (22) can be made arbitrarily small by choosing $A$ large enough, and, hence,

$$
\lim _{A \rightarrow \infty} \limsup _{n \rightarrow \infty} \mathrm{P}\left(X_{3}^{A *} \neq 0\right)=0
$$

and that $\mu_{A} \rightarrow \mu=\frac{1}{6}\left(b \mathrm{E}\left(W^{2}\right)\right)^{3}$ as $A \rightarrow \infty$. It follows by a standard argument (see [3, Theorem 4.2]) that we can let $A \rightarrow \infty$ in (23) and obtain

$$
X_{3} \stackrel{\mathrm{D}}{\rightarrow} \operatorname{Po}(\mu)
$$


In particular, $\mathrm{P}\left(X_{3}=0\right) \rightarrow \mathrm{e}^{-\mu}$, which yields

$$
\begin{aligned}
& \mathrm{P}(\omega(G(n, \alpha)) \leq 2) \rightarrow \mathrm{e}^{-\mu}=\mathrm{e}^{-\left(b \mathrm{E}\left(W^{2}\right)\right)^{3} / 6}, \\
& \mathrm{P}(\omega(G(n, \alpha))=3) \rightarrow 1-\mathrm{e}^{-\mu}=1-\mathrm{e}^{-\left(b \mathrm{E}\left(W^{2}\right)\right)^{3} / 6} .
\end{aligned}
$$

Finally, note that $G(n, \alpha)$ contains, w.h.p., cliques $K_{2}$, i.e. edges, so, clearly, w.h.p.,

$$
\omega(G(n, \alpha)) \geq 2
$$

\section{Final remarks}

In this section we make some comments on other models of power-law random graphs as well as some remarks on possible variants of our results. We omit detailed proofs.

Let us remark first that, for convenience and to facilitate comparisons with other papers, in the definition of $G(n, \alpha)$ we used two scale parameters, $a$ and $b$, above, besides the important exponent $\alpha$. By rescaling $W_{i} \mapsto t W_{i}$ for some fixed $t>0$ we obtain the same $G(n, \alpha)$ for the parameters $a t^{\alpha}$ and $b t^{-2}$; hence, only the combination $a b^{\alpha / 2}$ matters, and we could fix either $a$ or $b$ as 1 without loss of generality.

\subsection{Algorithms based on degrees}

In order to define $\mathcal{K}_{\mathrm{gr}}, \mathcal{K}_{\mathrm{qt}}$, and $\mathcal{K}_{\mathrm{ft}}$, we ordered the vertices of $G(n, \alpha)$ by their weights and examined them one by one. However, we could also order them by their degrees (taking the degrees of either the multigraph $\hat{G}(n, \alpha)$ or the corresponding simple graph), and define $\tilde{\mathcal{K}}_{\mathrm{gr}}, \tilde{\mathcal{K}}_{\mathrm{qt}}$, and $\tilde{\mathcal{K}}_{\mathrm{ft}}$ accordingly. It turns out that Theorem 2 remains true if we replace $\mathcal{K}_{\mathrm{gr}}$, $\mathcal{K}_{\mathrm{qt}}$, and $\mathcal{K}_{\mathrm{ft}}$, by $\tilde{\mathcal{K}}_{\mathrm{gr}}, \tilde{\mathcal{K}}_{\mathrm{qt}}$, and $\tilde{\mathcal{K}}_{\mathrm{ft}}$, respectively. The reason is that, for the vertices of large weight that we are interested in, the degrees are, w.h.p., all almost proportional to the weights, and, thus, the two orders do not differ very much. This enables us to find an almost maximal clique in polynomial time, even without knowing the weights.

\subsection{More general weight distributions}

Observe that Theorems 1 and 2 remain true (and can be shown by basically the same argument), provided that only the power law holds asymptotically for large weights, i.e. (1) may be relaxed to

$$
\mathrm{P}(W>x) \sim a x^{-\alpha} \text { as } x \rightarrow \infty .
$$

\subsection{Deterministic weights}

Instead of choosing weights independently according to the distribution $W$ we may as well take a suitable deterministic sequence $W_{i}$ of weights (as in [7]), for example,

$$
W_{i}=a^{1 / \alpha} \frac{n^{1 / \alpha}}{i^{1 / \alpha}}, \quad i=1, \ldots, n .
$$

All our results remain true in this setting also; in fact, the proofs are slightly simpler for this model. A particularly interesting special case for this model (see [5, Section 16.2] and [15]) is when $\alpha=2$, where (3) and (25) combine to yield

$$
\lambda_{i j}=\frac{a b}{\sqrt{i j}} .
$$




\subsection{Poisson number of vertices}

We may also let the number of vertices be random with a Poisson $\operatorname{Po}(n)$ distribution (as in, e.g. [13]). Then the set of weights $\left\{W_{i}\right\}_{1}^{n}$ can be regarded as a Poisson process on $[0, \infty)$ with intensity measure $n \mathrm{~d} \mu$, where $\mu$ is the distribution of the random variable $W$ in (1). Note that now $n$ can be any positive real number.

\subsection{Different normalization}

A slightly different power-law random graph model emerges when instead of (3) we define the intensities $\lambda_{i j}$ by

$$
\lambda_{i j}=\frac{W_{i} W_{j}}{\sum_{k=1}^{n} W_{k}}
$$

(see, e.g. [7] and [14]). Let us call this model $\tilde{G}(n, \alpha)$. In the case $\alpha>1$, when the mean E $W<\infty$, the results for $\tilde{G}(n, \alpha)$ and $G(n, \alpha)$ are not much different. In fact, by the law of large numbers, $\sum_{1}^{n} W_{k} / n \rightarrow \mathrm{E} W$ almost surely, so we may, for any $\varepsilon>0$, couple $\tilde{G}(n, \alpha)$ constructed by this model with $G(n, \alpha)^{ \pm}$constructed as above, using (3) with $b=1 /(\mathrm{E} W \mp \varepsilon)$, such that, w.h.p., $G(n, \alpha)^{-} \subseteq \tilde{G}(n, \alpha) \subseteq G(n, \alpha)^{+}$, and it follows that we have the same asymptotic results as in our theorems if we let $b=1 / \mathrm{E} W$.

On the other hand,

$$
\sum_{1}^{n} W_{k}=\left(a+o_{\mathrm{p}}(1)\right) n \log n \quad \text { for } \alpha=1
$$

and

$$
\sum_{1}^{n} \frac{W_{k}}{n^{1 / \alpha}} \stackrel{\mathrm{D}}{\rightarrow} Y \quad \text { for } 0<\alpha<1,
$$

where $Y$ is a stable distribution with exponent $\alpha$ (see, e.g. [9, Section XVII.5]). It follows, arguing as in Section 3 but using the random cutoffs $(1 \pm \varepsilon)\left(\frac{1}{2} \sum_{1}^{n} W_{k} \log n\right)^{1 / 2}$, that the size of the largest clique is always close to $\sqrt{n}$ for $\alpha \leq 1$; more precisely, for $\alpha=1$ (when we can use the equivalent deterministic cutoffs $(1 \pm \varepsilon) \sqrt{a n / 2} \log n)$, the largest clique in $\tilde{G}(n, \alpha)$ has

$$
\frac{\left(1+o_{\mathrm{p}}(1)\right) \sqrt{2 a n}}{\log n}
$$

vertices, while, for $0<\alpha<1$,

$$
\frac{\omega(\tilde{G}(n, \alpha))}{\sqrt{n} \log ^{-\alpha / 2} n} \stackrel{\mathrm{D}}{\rightarrow} Z=a 2^{\alpha / 2} Y^{-\alpha / 2},
$$

where $Z$ is an absolutely continuous random variable whose distribution has the entire positive real axis as support. (The square $Z^{2}$ has, apart from a scale factor, a Mittag-Leffler distribution with parameter $\alpha$; see [4, Section 8.0.5].) Thus, for $\alpha<1, \omega(\tilde{G}(n, \alpha))$ is not sharply concentrated around its median; this is caused by the fact that the normalizing factor $\sum_{i} W_{i}$ is determined by its first terms which, clearly, are not sharply concentrated around their medians as well. Interestingly enough, since in the proof of Theorem 2 we dealt mostly with the probability space where we conditioned on $W_{i}$, the analogue of Theorem 2 holds for this model as well. Thus, for instance, despite the fact that neither the largest clique nor the full top clique are sharply concentrated in this model, we can show the sharp concentration result for the ratio of these two variables. 


\subsection{The model $\min \left(\lambda_{i j}, 1\right)$}

For small $\lambda_{i j}$, (4) implies that $p_{i j} \approx \lambda_{i j}$. In most works on inhomogeneous random graphs, it does not matter whether we use (4) or, for example, $p_{i j}=\min \left(\lambda_{i j}, 1\right)$ or $p_{i j}=\lambda_{i j} /\left(1+\lambda_{i j}\right)$ (as in [6]); see [5]. For the cliques studied here, however, what matters is mainly the probabilities $p_{i j}$ that are close to 1 , and the precise size of $1-p_{i j}$ for them is important; thus, it is important that we use (4) (cf. [1] and [2], where a cutoff is introduced). For instance, a common version (see, e.g. [5]) of $G(n, \alpha)$ replaces (4) by

$$
p_{i j}=\min \left(\lambda_{i j}, 1\right) .
$$

This makes very little difference when $\lambda_{i j}$ is small, which is the case for most $i$ and $j$, and, for many asymptotical properties, the two versions are equivalent (see, again, [5]). In the case $\sum_{i} W_{i}^{3}=o_{\mathrm{p}}\left(n^{3 / 2}\right)$, which in our case with $W_{i}$ governed by (1) holds for $\alpha>2$ as a consequence of (19), a strong general form of asymptotic equivalence is proved in [11]; in the case $\alpha=2$, when $\sum_{i} W_{i}^{3}=o_{\mathrm{p}}\left(n^{3 / 2}\right)$, by (17), a somewhat weaker form of equivalence (known as contiguity) holds provided that, say, $\max _{i j} \lambda_{i j} \leq 0.9$ also; see again [11]. In our case we do not need these general equivalence results; the proofs above for the $\alpha \geq 2$ cases hold for this model too, so Theorem 1(ii) and (iii) hold without changes.

If $\alpha<2$, however, the results are different. In fact, (26) implies that all vertices with $W_{i} \geq b^{-1 / 2} n^{1 / 2}$ are joined to each other, and, thus, form a clique; conversely, if we now define $V^{-}=\left\{i: W_{i} \leq(b+\varepsilon)^{-1 / 2} n^{1 / 2}\right\}$ then $p_{i j}=\lambda_{i j} \leq b /(b+\varepsilon)$ for $i, j \in V^{-}$, and, thus, $\omega\left(G(n, \alpha)\left[V^{-}\right]\right)=O(\log n)$ w.h.p. by Lemma 1. Consequently, arguing as in Section 3,

$$
\omega(G(n, \alpha))=\left(1+o_{\mathrm{p}}(1)\right) n \mathrm{P}\left(W>b^{-1 / 2} n^{1 / 2}\right)=\left(1+o_{\mathrm{p}}(1)\right) a b^{\alpha / 2} n^{1-\alpha / 2},
$$

so the logarithmic factor in Theorem 1(i) disappears.

\subsection{The model $\lambda_{i j} /\left(1+\lambda_{i j}\right)$}

Another version of $G(n, \alpha)$ replaces (4) by

$$
p_{i j}=\frac{\lambda_{i j}}{1+\lambda_{i j}} .
$$

This version has the interesting feature that, conditioned on the vertex degrees, the distribution is uniform over all graphs with that degree sequence; see [6].

In this version, for large $\lambda_{i j}, 1-p_{i j}=1 /\left(1+\lambda_{i j}\right)$ is considerably larger than for (4) (or (26)), and, as a consequence, the clique number is smaller. For $\alpha \geq 2$, stochastic domination (or a repetition of the proofs above) shows that Theorem 1(ii) and (iii) hold without changes.

For $\alpha<2$, there is a significant difference. Arguing as in Section 3, we find that, for some constants $c$ and $C$ depending on $a, b$, and $\alpha$, w.h.p.,

$$
c n^{(2-\alpha) /(2+\alpha)} \leq \omega(G(n, \alpha)) \leq C n^{(2-\alpha) /(2+\alpha)}(\log n)^{\alpha /(2+\alpha)} .
$$

Although this only determines the clique number up to a logarithmic factor, note that the exponent of $n$ is $(2-\alpha) /(2+\alpha)$, which is strictly less than the exponent $(2-\alpha) / 2$ in Theorem 1 .

\subsection{Preferential attachment}

Finally, let us observe that not all power-law random graph models contain large cliques. Indeed, one of the most popular types of model of such graphs is the preferential attachment 
graph in which the graph grows by acquiring new vertices, where each new vertex $v$ is joined to some number $k_{v}$ of 'old' vertices according to some random rule (which usually depends on the structure of the graph we have constructed so far); see, e.g. [8]. Clearly, such a graph on $n$ vertices cannot have cliques larger than $X_{n}=\max _{v \leq n} k_{v}+1$, and since, for most of the models, $X_{n}$ is bounded from above by an absolute constant or grows very slowly with $n$, typically the size of the largest clique in preferential attachment random graphs is small.

\section{Acknowledgements}

We thank an anonymous referee for valuable comments. This research was carried out at Institut Mittag-Leffler, Djursholm, Sweden, during the program 'Discrete Probability' 2009. The second author was partially supported by the Foundation for Polish Science.

\section{References}

[1] Bianconi, G. And Marsili, M. (2006). Emergence of large cliques in random scale-free networks. Europhys. Lett. 74, 740-746.

[2] Bianconi, G. and Marsili, M. (2006). Number of cliques in random scale-free network ensembles. Physica D 224, 1-6.

[3] Billingsley, P. (1968). Convergence of Probability Measures. John Wiley, New York.

[4] Bingham, N. H., Goldie, C. M. And Teugels, J. L. (1987). Regular Variation. Cambridge University Press.

[5] Bollobás, B., Janson, S. And Riordan, O. (2007). The phase transition in inhomogeneous random graphs. Random Structures Algorithms 31, 3-122.

[6] Britton, T., Deijfen, M. and Martin-LöF, A. (2006). Generating simple random graphs with prescribed degree distribution. J. Statist. Phys. 124, 1377-1397.

[7] Chung, F. And Lu, L. (2002). Connected components in random graphs with given expected degree sequences. Ann. Combinatorics 6, 125-145.

[8] Durrett, R. (2007). Random Graphs Dynamics. Cambridge University Press.

[9] Feller, W. (1971). An Introduction to Probability Theory and Its Applications, Vol. II, 2nd edn. John Wiley, New York.

[10] Frieze, A. AND McDiarmid, C. (1997). Algorithmic theory of random graphs. Random Structures Algorithms 10, 5-42.

[11] Janson, S. (2008). Asymptotic equivalence and contiguity of some random graphs. Preprint. Available at http://arxiv.org/abs/0802.1637v1.

[12] Janson, S., ŁuCZaK, T. And Ruciński, A. (2000). Random Graphs. Wiley-Interscience, New York.

[13] Norros, I. (2009). A mean-field approach to some Internet-like random networks. In Proc. 21st Internat. Teletraffic Congress (Paris, September 2009), North-Holland, Amsterdam, pp. 1-8.

[14] Norros, I. And Reittu, H. (2006). On a conditionally Poissonian graph process. Adv. Appl. Prob. 38, 59-75.

[15] Riordan, O. (2005). The small giant component in scale-free random graphs. Combinatorics Prob. Comput. 14, 897-938.

[16] Van der Hofstad, R., Hooghiemstra, G. and Znamenski, D. (2006). Distances in random graphs with infinite mean degrees. Extremes 8, 111-141. 